\title{
Telephone-based interventions in outpatient care
}

Telephone calls have long been integral to health care delivery. Patients often contact physicians to determine whether symptoms require a face to face visit, and physicians call patients to follow up office visits. As it became increasingly clear that patient education for chronic disease requires time beyond a single office visit, telephone-based intervention became viewed as a potential effective way to overcome pragmatic obstacles (for example, space, time) to educate patients in already busy outpatient settings. Telephone contacts also provide an excellent vehicle through which to monitor patients' health between office visits, particularly when patients need to travel long distances to receive medical care.

More than a decade ago, we began to examine the impact of proactively using telephone-based interventions for patients with osteoarthritis (OA). An uncontrolled, longitudinal study observed that, following biweekly telephone calls during a six month period, patients with OA reported significant improvements in functional status. ${ }^{1}$ Perhaps more surprisingly, six months after telephone calls were ceased, improvements in functional status persisted. ${ }^{1}$

Unfortunately, this investigation lacked a control group. Thus, we conducted a randomised controlled trial to evaluate whether telephone-based or office-based interventions, or both, improved functional status of patients with OA. The intervention consisted of brief interviews during which trained non-medical personnel reviewed patients: (1) medications, (2) joint pain symptoms, (3) gastrointestinal complaints related to use of non-steroidal antiinflammatory drugs, (4) reporting of early warning signs of hypertension, heart disease, diabetes, chronic obstructive pulmonary disease (when applicable), (5) scheduled outpatient visits, (6) an existing process by which patients could telephone a provider during evenings and weekends, and (7) barriers to keeping their appointments. These personnel also provided specific suggestions to encourage patients to ask questions of their providers. At one year, compared with the control group, persons receiving telephone contacts reported significantly less physical disability and pain, as well as a trend towards better psychological status. ${ }^{2}{ }^{3}$ Interestingly, patients receiving the office-based intervention had worse physical health, although neither pain nor psychological health was affected. The latter finding provides additional support for delivering such interventions by telephone, instead of during regularly scheduled primary care visits. The telephone intervention was relatively inexpensive to deliver, ${ }^{4}$ yet reaped clinical benefits in the range achieved during trials of drug therapy for OA.

Subsequently, other investigators have evaluated telephone-based interventions among patients with rheumatological problems. One randomised trial compared two telephone-based strategies (symptom monitoring, treatment counselling) against usual care among patients with $\mathrm{OA}$ and rheumatoid arthritis (RA). The two intervention strategies differed in that treatment counselling was more elaborate and was multi-faceted. The authors found that treatment counselling had a clear advantage over either symptom monitoring or usual care, ${ }^{5}$ a finding that was also seen among patients with systemic lupus erythematosus (SLE). ${ }^{6}$ In addition, an early study suggests that person centred counselling, which focuses on the affective state of the person (including coping with emotional stress), may be effective among persons with SLE, although not in RA. ${ }^{7}$
Outside rheumatology, Wasson et al used a randomised controlled trial to evaluate whether telephone care could substitute for office visits. ${ }^{8}$ For patients in the intervention group, clinicians' recommended follow up visit intervals were doubled (for example, from three to six months), and three intervening telephone calls were substituted. Compared with patients in the control group, patients in the intervention group had significantly fewer office visits, less medication use, fewer hospital admissions, shorter stays in the hospital (both wards and intensive care units), and health care expenditures. Notably, for patients reporting fair or poor overall health at baseline ( $36 \%$ of the sample), there was a trend $(p=0.06)$ towards an advantage in survival for the intervention group. This study shows the potent effects of telephone-based interventions.

\section{Future applications of telephone-based programmes}

While randomised trials have shown the effectiveness of telephone-based interventions, several issues are likely to affect the future application of this promising modality in clinical practice.

Firstly, how can patients effectively provide requisite data to their physician, and how can the physician be trained to use these data? For example, what if patients could transmit relevant serial data to their physician from their homes - that is, without requiring a face to face office visit? In a study of patients with insulin dependent diabetes mellitus, Marrero et al evaluated a technology that allowed patients to download their daily blood glucose values to their providers via modem. Providers could then display these data to detect patterns of hypoglycaemia and hyperglycaemia and use these data to adjust patients' regimens. The investigators found that, although patients enjoyed using this technology, its use had no effect on glycaemic control. ${ }^{9}$ An important question in rheumatology is whether the health of arthritis patients could be improved by providing data about their functional status to their physician. While intuitively appealing, two separate randomised controls trial found that simply educating physicians about the importance of their patients' functional status and providing them with specific functional status data had no impact upon patients' health. ${ }^{11}{ }^{11}$ The authors conclude that physicians will need additional training on how to interpret and act upon their patients' functional status scores.

Secondly, both the cost and effectiveness of telephonebased interventions is affected by who delivers the intervention (for example, physician, nurse, lay person). Maisiak and his colleagues found that counsellors trained in specific protocols can improve outcomes of patients with rheumatic conditions; however, that improvement was not uniform across various rheumatic conditions..$^{5-7}$ Moreover, in another chronic disease model requiring complex regimens, diabetes mellitus, we examined the impact of a nurse coordinated intervention. Nurse initiated contacts were made by telephone between office visits at least monthly to provide patient education, reinforce compliance with regimens, monitor patients' health status, facilitate resolution of identified problems, and facilitate access to primary care. At one year, between group differences favoured intervention patients for glycaemic control; however, there was no effect on health related quality of life or diabetes related symptoms. ${ }^{12}$

A third consideration is how to efficiently reach patients who are difficult to contact by telephone. For patients with 
telephones, automated voice messaging (AVM) is a promising solution. Again, using diabetes mellitus as a model, Piette and Mah have developed a specialised computer technology designed to telephone patients at different times of the day until contact is made. ${ }^{13}$ Their AVM system both communicates messages and collects information, as patients respond to AVM questions via a touch-tone telephone. Their AVM system prompts patients for information about their symptoms, glucose monitoring, foot care, and medication adherence. Patients are also able to use AVM to select relevant health promotion messages if they desire. The investigators have developed a safeguard so that patients requiring immediate attention were called by a clinician. Initial studies suggest that the AVM system was extremely well received by patients. It is currently being evaluated using a randomised trial to examine whether patient outcomes are improved. Patients without telephones present a more difficult problem. There is no way to provide them with telephone-based interventions. Unfortunately, such patients may be those who might most benefit from such intervention, for example, if they are socioeconomically vulnerable patients, reside in rural areas, or have the greatest distance to travel for care.

Finally, who should pay for this service? When conducting any cost analyses, the perspective (for example, societal, health care system, patient) must be considered when answering such a question. Perhaps as health care systems are increasingly reimbursed using capitation (for example, managed care), issues faced by fee for service models will become less mettlesome.

\section{Conclusions}

Telephone-based interventions remain an exciting strategy to augment patient education, monitoring, and counselling programmes delivered through office-based programmes. The programmes reported are very sensitive to pragmatic issues faced in the outpatient setting, for example, time and space constraints. Moreover, with the nearly ubiquitous use of the internet and world wide web, as well as rapidly developing technologies, there may be increased opportunities for creative ways in which to use telephone-based interventions to improve patient outcomes and increase the efficiency of health care.

MORRIS WEINBERGER

Health Services Research (11H), Roudebush VAMC, 1481 West Tenth

Street, Indianapolis, IN 46202, USA

Regenstrief Institute for Health Care, Department of Medicine,

Indiana University School of Medicine

1 Weinberger M, Hiner SL, Tierney WM. Improving functional status in arthritis: the effect of social support. Soc Sci Med 1986;23:899-904.

2 Weinberger M, Tierney WM, Booher P, Katz BP. Can the provision of information to patients with osteoarthritis improve functional status: a mation to patients with osteoarthritis improve functional

3 Rene J, Weinberger M, Mazzuca SA, Brandt KD, Katz BP. Reduction of joint pain in patients with knee osteoarthritis who have received monthly telephone calls from lay personnel and whose medical treatment regimens have remained stable. Arthritis Rheum 1992;35:511-15.

4 Weinberger M, Tierney WM, Cowper PA, Katz BP, Booher P. Costeffectiveness of increased telephone contact for patients with osteoarthritis: a randomized, controlled trial. Arthritis Rheum 1993;36:243-6.

5 Maisiak R, Austin J, Heck L. Health outcomes of two telephone interventions for patients with rheumatoid arthritis or osteoarthritis. Arthritis Rheum 1996;39:1391-9.

6 Austin JS, Maisiak RS, Macrina DM, Heck LW. Health outcome improvements in patients with systemic lupus erythematosus using two telephone counseling interventions. Arthritis Care Res 1996;9:391-9.

7 Maisiak R, Austin JS, West SG, Heck L. The effect of person-centered counseling on the psychological status of persons with systemic lupus erythematosus or rheumatoid arthritis: a randomized, controlled trial. Arthrithematosus or rheumatoid
tis Care Res 1996;9:60-6.

8 Wasson J, Gaudette C, Whaley F, Sauvigne A, Baribeau P, Welch HG. Telephone care as a substitute for routine clinic follow-up. JAMA ephone care as a
$1992 ; 267: 1788-93$.

9 Marrero DG, Vandagriff J, Kronz K, Fineberg NS, Golden MP, Gray D, et al. Using telecommunication technology to manage children with diabetes: the computer-linked outpatient clinic (CLOC) study. Diabetes Educator 1995;21:313-19.

10 Rubenstein LV, Calkins DR, Young RT, Cleary PD, Fink A, Kosecoff J, et al. Improving patient function: a randomized trial of functional disability screening. Ann Intern Med 1989;11:836-42.

11 Calkins DR, Rubenstein LV, Cleary PD, Davies AR, Jette AM, Fink A, et al. Functional disability screening of ambulatory patients: a randomized controlled trial in a hospital-based group practice. J Gen Intern Med 1994;9: 590-2.

12 Weinberger M, Kirkman MS, Samsa GP, Cowper PA, Shortliffe EA, Simel $\mathrm{DL}$, et al. A nurse-coordinated intervention for primary care patients with no, et al. A nurse-coordinated intervention for primary care patients with health-related quality of life. J Gen Intern Med 1995;10:59-66.

13 Piette JD, Mah CA. The feasibility of automated voice messaging as an adjunct to diabetes outpatient care. Diabetes Care 1997;20:15-21. 